\title{
Improvements in MR Angiography Using Phase-Corrected Data Sets
}

\author{
Thomas L. Chenevert, Klaus P. Fechner, and Daphna Y. Gelblum \\ Department of Radiology, University of Michigan Medical Center, Ann Arbor, Michigan 48109
}

Received April 20, 1988; revised September 21, 1988

\begin{abstract}
The objective of this work is refinement of an MR angiography technique via postprocessing removal of phase errors which inhibit static signal subtraction. Projective views of the object are obtained using interleaved flow-compensated and noncompensated gradient waveforms. Complex subtraction of data sets is required since a projection dephase pulse is used for static signal suppression. This renders the difference image susceptible to systematic phase errors which are modeled as a smoothly varying multiplicative phase function. The error function is estimated by comparison of heavily spatial filtered renditions of the object acquired without projection dephasing in order to minimize influence of flow. The phase correction is then applied to high-resolution data sets collected with projection dephasing to enhance flow sensitivity. The technique is demonstrated by improvement of MR angiograms of rats acquired on a 2-T, 31-cm-bore system. (c) 1989 Academic Press, Inc.
\end{abstract}

\section{INTRODUCTION}

Tissue dynamics are among the properties to which magnetic resonance (MR) imaging is sensitive. Often dynamics are secondary to the objective of the MR imaging experiment and may either enhance or detract from the diagnostic value of the image. This is particularly true for acquisition sequences designed without special consideration of motion $(1,2)$. A variety of techniques has been developed to attempt blood flow quantification or direct imaging of flow patterns. Usually these are categorized into methods based on amplitude modulation due to bulk motion of spins through selected volumes (3-5) or methods which exploit phase shifts that are unique to flow $(6-8)$. Within each category there is a variety of flow information representations such as phase displays, angiograms, and cinematography. Our interests lie in projective MR angiography.

Several MR angiography schemes rely on subtraction of images which, ideally, differ only in flow signal. Flow is distinguished from static tissue by its pulsatile nature (9) or by gradient waveforms which induce phase shifts unique to motion (10-12). When the angiographic method necessitates complex subtraction of data sets, technical imperfections may compromise the final result. For example, gradient eddy current effects are a notorious source of phase errors which confound complex subtraction angiograms (11).

The objective of our study is refinement of an MR projection angiography technique. This particular technique does not attempt to quantify flow speed or direction; rather it strives to display flow patterns with minimal dependence on speed and ulti- 
mately may be expanded to be independent of flow direction. Consequently, cardiac gating is not required nor must the flow be pulsatile since gradient waveforms, not cardiac phase, are used to distinguish static from flow. It is assumed, however, that flow speed is adequate to be distinguished from static for the given gradient strength and duration. Pulsatile flow having a static phase is a source of motion artifact which can be mitigated by signal averaging or gating.

In its simplest form this angiographic process uses subtraction of magnitude images (11), thereby circumventing many technical imperfections apparent in complex data. However, substantial improvement in signal to noise may be realized via projection dephasing gradient pulses to suppress static signal (10). Inclusion of projection dephasing requires complex subtraction of data sets to avoid yet additional artifacts unrelated to technical errors. Consequently, phase equivalence between data sets is essential otherwise flow patterns may be masked by incomplete subtraction of static signal. In the following report we present a simple phase correction scheme to improve complex subtraction MR angiography. While demonstrated here with flow speed insensitive angiography, the approach may also benefit other phase-dependent flow imaging/quantification methods.

\section{MATERIALS AND METHODS}

Phase-sensitive flow imaging strategies exploit the fact that motion in the presence of a gradient produces additional phase shifts. In general, the phase of a spin traveling path $\mathbf{r}(t)$ through a time-varying gradient $\mathbf{G}(t)$ is given by

$$
\Phi=\gamma \int \mathbf{G}(t) \cdot \mathbf{r}(t) d t
$$

The function $\mathbf{r}(t)$ may be expanded into initial location, velocity, acceleration, and higher order terms so that the corresponding phase shifts are easily calculable for a given $\mathbf{G}(t)(13)$. Alternatively, one may prescribe desired motion dependencies of $\Phi$ as design criteria for $\mathbf{G}(t)(14)$. We use two gradient waveforms to produce angiograms with minimal flow speed dependence. In one, $\mathbf{G}(t)$ induces strong velocitydependent phase shifts such that even in relatively slow flow there is sufficient phase dispersion to attenuate the flow signal. The other waveform should produce minimal velocity-dependent phase shifts thereby refocusing flow into a strong coherent signal. Short echo time helps to minimize acceleration and higher order effects which are ignored. Flow direction sensitivity is determined by $\mathbf{G}(t)$ which may represent any one or combination of the three gradient directions; frequency encoding, phase encoding, and projection. All flow-compensated or noncompensated gradients can be applied simultaneously for omnidirectional flow sensitivity. In this report, however, flow sensitivity is limited to only the frequency-encoding direction.

Figure 1 shows frequency-encoding gradient waveforms (a) $G_{\mathrm{NC}}$ which produce a velocity-dependent phase shift (flow noncompensated) and (b) $G_{\mathrm{FC}}$ which produces no velocity-dependent phase shifts (flow compensated). Forcing zeroth and first moments of $\mathbf{G}(t)$ to be zero and solving for gradient amplitudes $G_{\mathrm{A}}$ and $G_{\mathrm{B}}$ in terms of observe amplitude $G_{\mathrm{C}}$ and timing parameters one finds 


$$
\begin{aligned}
G_{\mathrm{A}} & =G_{\mathrm{C}} t_{1}\left[t_{1} / 2+\tau / 2-t_{2}\right] /\left[\tau\left(t_{2}-t_{3}\right)\right] \\
G_{\mathrm{B}} & =G_{\mathrm{C}} t_{1}\left[t_{1} / 2+\tau / 2-t_{3}\right] /\left[\tau\left(t_{2}-t_{3}\right)\right] .
\end{aligned}
$$

There is a continuum of waveform designs which have the desired flow dependencies; however, we were driven by the empiric observation that eddy current effects were reduced by minimizing gradient amplitude excursions. According to Eq. [2] this is achieved by maximizing the temporal separation of pulses $G_{\mathrm{A}}$ and $G_{\mathrm{B}}$. The waveform $G_{\mathrm{NC}}$ differs from conventional spin-echo design only in that its observe pulse duration is increased to amplify flow phase shift.

Proceeding from the assumption that flow signal is sufficiently attenuated by $G_{\mathrm{NC}}$ and recovered by $G_{\mathrm{FC}}$, the respective complex projections along axis $z$ may be modeled as

$$
\begin{aligned}
P_{\mathrm{NC}}(x, y) & =\int s(x, y, z) d z=S(x, y) \\
P_{\mathrm{FC}}(x, y) & =\int[s(x, y, z)+f(x, y, z)] e^{i \delta(x, y)} d z \\
& =[S(x, y)+F(x, y)] e^{i \delta(x, y)},
\end{aligned}
$$

where $S$ is the projection of static tissues, $F$ is the projection of flow, and $\delta(x, y)$ represents any phase difference that may exist between data sets. In particular, we attribute the majority of phase differences to eddy current differences between the sequences. Implicit in this model are assumptions that there is no significant phase error dependence on $z$ since the $z$ gradient is unchanged and that eddy current differences are negligible during signal digitization, otherwise Eq. [3] would have a convolutional form.

Within this model it is obvious that magnitude subtraction of $\left|P_{\mathrm{NC}}\right|$ from $\left|P_{\mathrm{FC}}\right|$ produces an angiogram free of phase error. Unfortunately, in most situations flow contributes relatively little to $P_{\mathrm{FC}}$. Due to dynamic range limits of typical MRI systems, subtraction-based flow imaging schemes can suffer quantization noise problems when the receiver gain setting is dominated by the static signal. Moreover, minor imperfections in sequence execution which result in incomplete subtraction of static signal may completely mask flow. This inherent low flow sensitivity due to static signal constitutes another major impediment to MR angiography based on image differences. A simple and effective static signal suppression technique is inclusion of a spin dephasing gradient pulse along the projection axis direction (10). With the application of a projection dephasing gradient pulse of a strength $\lambda \mathrm{rad} / \mathrm{mm}$ the complex data may be modeled as

$$
\begin{aligned}
D_{\mathrm{NC}}(x, y) & =\int s(x, y, z) e^{i \lambda z} d z=S_{\lambda}(x, y) \\
D_{\mathrm{FC}}(x, y) & =\int[s(x, y, z)+f(x, y, z)] e^{i \delta(x, y)} e^{i \lambda z} d z \\
& =\left[S_{\lambda}+F_{\lambda}\right] e^{i \delta(x, y)} .
\end{aligned}
$$

The functions $S_{\lambda}$ and $F_{\lambda}$ represent the $z$ direction spatial frequency content of static 


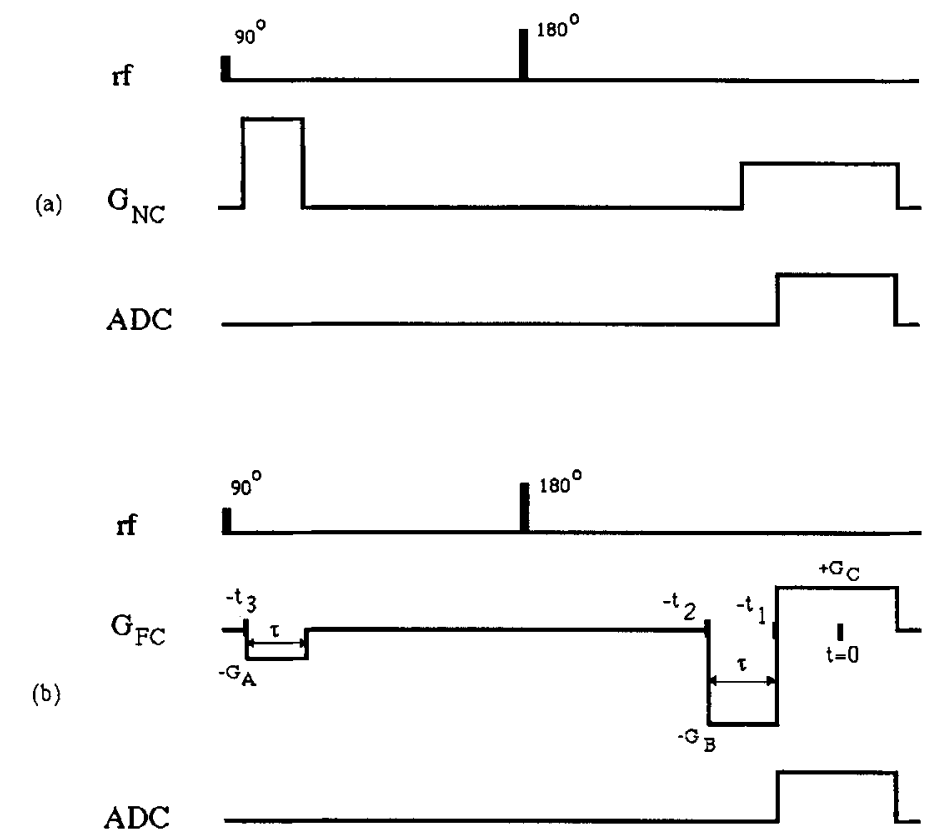

FIG. 1. Frequency-encoding gradient waveforms. (a) Waveform $G_{\mathrm{NC}}$ is not compensated for flow and therefore attenuates flow signal due to dephasing. (b) Waveform $G_{\mathrm{FC}}$ is flow compensated and recovers flow signal.

and flow objects at the spatial frequency defined by $\lambda$. If the dimension of vessels in the projection direction is small compared to static tissues, as is usually the case, the relative contribution of flow to the recorded signal will be enhanced. For example, if one considers the simplest case of a uniform object having static tissue thickness $t_{\mathrm{S}}$ and vessel thickness $t_{\mathrm{F}}$ and ignores relaxation time differences and other physical details, the ratio of flow to static contributions to $D_{\mathrm{FC}}$ is given by

$$
F_{\lambda} / S_{\lambda}=\left|t_{\mathrm{F}} \operatorname{sinc}\left(t_{\mathrm{F}} \lambda / 2\right)\right| /\left|t_{\mathrm{S}} \operatorname{sinc}\left(t_{\mathrm{S}} \lambda / 2\right)\right| \text {. }
$$

For $t_{\mathrm{S}} \gg t_{\mathrm{F}}$ and $t_{\mathrm{F}} \lambda<\pi / 2$ it is apparent that the flow contribution is significantly enhanced over the nonprojection dephased case in which the ratio is given by $t_{\mathrm{F}} / t_{\mathrm{S}}$.

The magnitude difference of flow-compensated and noncompensated data sets of Eq. [4] eliminates the function $\delta(x, y)$; however, the relative phase between $S_{\lambda}$ and $F_{\lambda}$ modulates the intensity of the angiogram. That is,

$$
\begin{aligned}
\left|D_{\mathrm{FC}}\right|-\left|D_{\mathrm{NC}}\right| & =\left|S_{\lambda}+F_{\lambda}\right|-\left|S_{\lambda}\right| \\
& =\left\{\left|S_{\lambda}\right|^{2}+\left|F_{\lambda}\right|^{2}+2\left|S_{\lambda} \| F_{\lambda}\right| \cos (\phi)\right\}^{1 / 2}-\left|S_{\lambda}\right|,
\end{aligned}
$$

where $\phi$ represents the relative phase between $S_{\lambda}$ and $F_{\lambda}$. By inspection of Eq. [4] it is clear that projection dephasing results in $\phi$ being a function $z$. Consequently, flow intensity in the angiogram is dependent on vessel position along the $z$ axis. In addi- 
tion, as the product of projection dephasing strength and vessel thickness approaches $2 \pi$, one can expect reduced vessel intensity as $F_{\lambda}$ approaches zero.

These artifacts are exemplified in the phantom images of Fig. 2. The phantom consists of a fluid-filled petri dish with a removable glass insert. Data collected with the insert removed simulate flow-compensated acquisition (i.e., no signal void), whereas data collected with the glass insert in place simulate flow void of an ideal noncompensated acquisition. Gradient waveforms were unchanged thereby avoiding eddy current complications in the simulation. To display the thickness-dependent artifact the glass insert was constructed of five glass slides $(1 \times 25 \times 75 \mathrm{~mm}$ each $)$ overlapped such that the net thickness with respect to the projection direction stairsteps from $1 \mathrm{~mm}$ at right and left edges to $5 \mathrm{~mm}$ in the middle. This thickness variation is along the horizontal direction of the images and is faithfully depicted in the images collected without projection dephasing (Figs. 2a magnitude and $2 b$ complex difference ), albeit at low signal to noise. To demonstrate the position-dependent artifact the glass insert was tilted such that its position with respect to the projection direction ramped by $12 \mathrm{~mm}$ from top to bottom. The strong intensity modulation along vertical lines of the magnitude difference image (Fig. $2 \mathrm{c}$ ) collected with $0.48 \pi$ / $\mathrm{mm}$ projection dephasing illustrates the artifact. This artifact is nearly completely removed by complex subtraction (Fig. $2 \mathrm{~d}$ ); however, as expected the thickness-dependent artifact seen as modulation along horizontal lines remains. Herein lies the greatest fault with projection dephasing: the potential for interference of signal within thick vessels or between superimposed vessels. Nevertheless, our approach is to use projection dephasing for its signal-to-noise benefits and then to apply regional phase corrections to the data prior to complex subtraction as described below.

Referring again to Eq. [3], $P_{\mathrm{NC}}$ and $P_{\mathrm{FC}}$ differ only in the flow signal content and any phase distortions resulting from improper sequence tuning or eddy currents. Although system dependent, typically these errors are spatially slowly varying functions. Vessel patterns in the body, on the other hand, are characterized by higher spatial detail. In this case, one may compare heavily spatial filtered versions of $P_{\mathrm{NC}}$ and $P_{\mathrm{FC}}$ as a measure of phase error. Let $P_{\mathrm{NC}}^{\prime}$ and $P_{\mathrm{FC}}^{\prime}$ be defined as the convolution of $P_{\mathrm{NC}}$ and $P_{\mathrm{FC}}$ with a spatial blur function $B(x, y)$. Assuming $\delta(x, y)$ is a slowly varying function over the range defined by $B(x, y)$ then

$$
P_{\mathrm{FC}}^{\prime}=P_{\mathrm{FC}} \otimes B=\left[(S+F) e^{i \delta(x, y)}\right] \otimes B \cong[(S+F) \otimes B] e^{i \delta(x, y)} .
$$

Furthermore, if the contribution of flow to $P_{\mathrm{FC}}$ is small, its contribution to $P_{\mathrm{FC}}$ may be ignored leading to a simple estimation of phase error,

$$
P_{\mathrm{FC}}^{\prime} \cong[S \otimes B] e^{i \delta(x, y)}=P_{\mathrm{NC}}^{\prime} e^{i \delta(x, y)} .
$$

To minimize flow signal contamination of the phase error estimation process, these data sets are acquired without projection dephasing. Separate data sets to which phase corrections are applied are acquired with projection dephasing. That is, the angiogram $A(x, y)$ is finally generated by complex subtraction,

$$
A(x, y)=\left|\hat{D}_{\mathrm{FC}}-D_{\mathrm{NC}}\right| \text {, }
$$



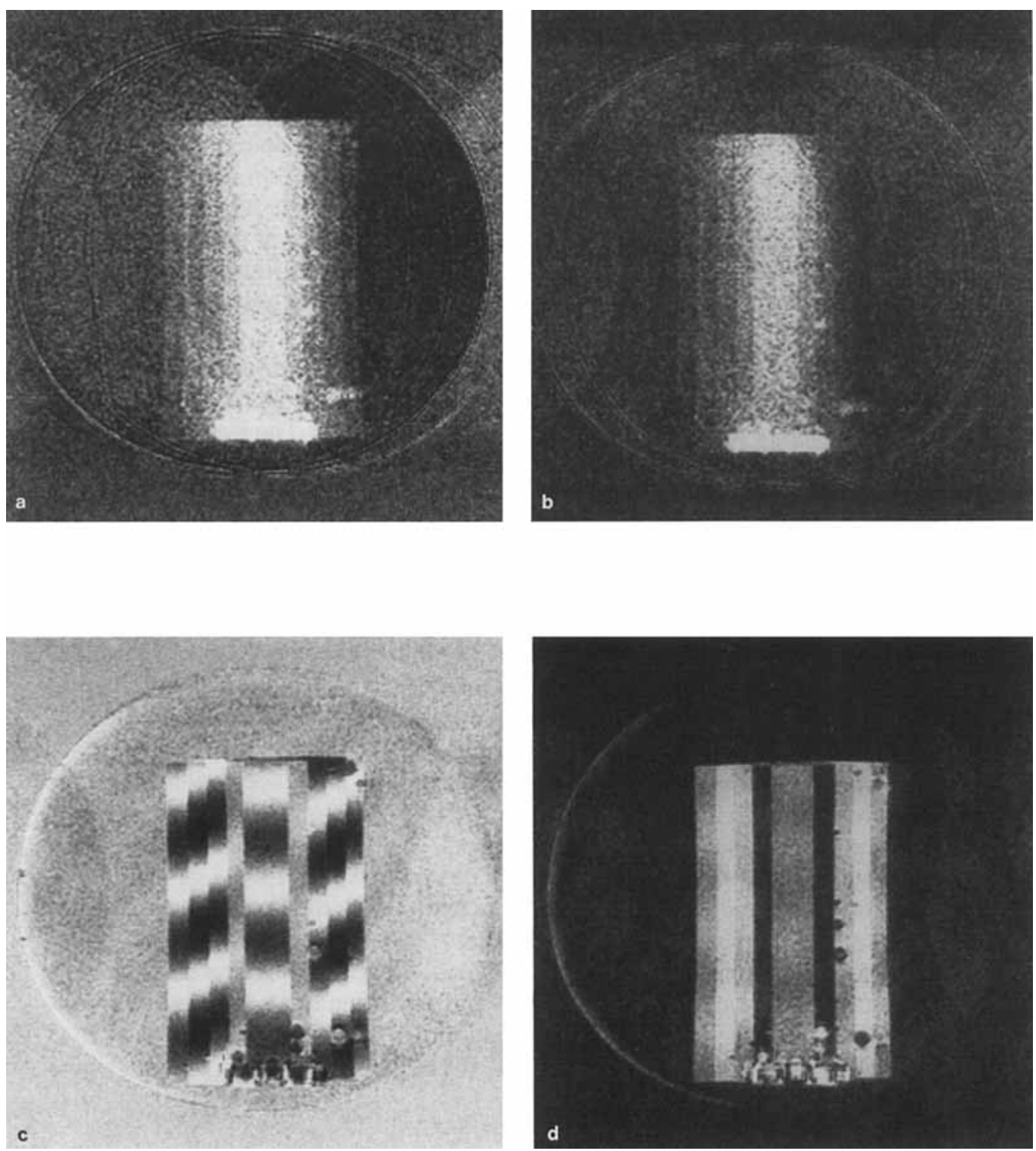

FIG. 2. Difference images of a phantom containing a removable glass slide insert to simulate flow-compensated and noncompensated acquisitions. Thickness of the insert varies from right to left, whereas its position with respect to the projection axis varies from top to bottom of the images. These images demonstrate vessel thickness and position-dependent artifacts in magnitude and complex difference images acquired with projection dephasing. (a) Magnitude difference image, acquired without projection dephasing. (b) Complex difference, without projection dephasing. (c) Magnitude difference, acquired with $0.48 \pi /$ mm projection dephasing. (d) Complex difference, acquired with $0.48 \pi / \mathrm{mm}$ projection dephasing. Note that only the position-dependent artifact (intensity modulation along vertical lines) is removed by complex subtraction. 
where

$$
\hat{D}_{\mathrm{FC}}=D_{\mathrm{FC}}\left[P_{\mathrm{NC}}^{\prime} P_{\mathrm{FC}}^{\prime *}\right] /\left[\left|P_{\mathrm{NC}}^{\prime}\right|\left|P_{\mathrm{FC}}^{\prime}\right|\right]
$$

and $P_{\mathrm{FC}}^{\prime *}$ is the complex conjugate of $P_{\mathrm{FC}}^{\prime}$.

The requirement for phase correction files represents only a marginal increase in total scan time. Since $P_{\mathrm{NC}}^{\prime}$ and $P_{\mathrm{FC}}^{\prime}$ are very low spatial resolution renditions of the object, only a sparse acquisition matrix (16-32) is required. We have adopted a 32 matrix size acquisition followed by heavy Gaussian apodization of the echoes to produce the phase correction files $P_{\mathrm{NC}}^{\prime}$ and $P_{\mathrm{FC}}^{\prime}$. Furthermore, as indicated in Eq. [9] knowledge of absolute phase is not required, thereby avoiding need of a phase unwrapping algorithm.

MR angiography was performed on rats using a 2-T, 31-cm-bore system (GE NMR Instruments). A two-turn surface coil receiver geometrically decoupled to a bird cage transmit coil was used to image the rat head and neck region. The frequency-encoding direction was selected as the long axis of the rat giving greatest flow sensitivity in the neck vessels.

Interleaving of flow-compensated and noncompensated acquisitions was used to minimize misregistration due to motion. Flow noncompensation and compensation were achieved via the waveforms of Fig. 1 with the minor modification of using 4-ms sine-shaped prephase pulses $\left(G_{\mathrm{A}}=4.9 \mathrm{mT} / \mathrm{m}, G_{\mathrm{B}}=18.2 \mathrm{mT} / \mathrm{m}\right)$ and ramps appending the observe gradient $\left(G_{\mathrm{c}}=10 \mathrm{mT} / \mathrm{m}\right)$. The noncompensated waveform produced a phase shift of approximately $0.6 \pi / \mathrm{cm} / \mathrm{s}$ of flow. The flow-compensated waveform promotes an undesired gradient echo from signal originating from the $180^{\circ}$ pulse; therefore, additional spoiler pulses were applied in the projection direction. Phase encoding immediately preceded signal digitization to reduce flow misregistration between phase- and frequency-encoding events. All acquisitions were ungated.

As described above, two pairs of flow-compensated and noncompensated data sets are required to produce an angiogram. One set consisted of interleaved flow-compensated and noncompensated $32 \times 256$ matrices acquired without projection dephasing. This provided the phase corrections to be applied to the other pair of $256 \times 256$ interleaved matrices collected with projection dephasing. Phase correction was performed in accordance with Eq. [9]. The spatial filtered functions $P_{\mathrm{NC}}^{\prime}$ and $P_{\mathrm{FC}}^{\prime}$ were generated by multiplication of the nonprojection dephased time domain data by a two-dimensional Gaussian function. The FWHM of the corresponding spatial Gaussian filter was 15 pixels.

\section{RESULTS AND DISCUSSION}

As a demonstration of the signal-to-noise improvement afforded by projection dephasing, Fig. 3 shows magnitude difference angiograms produced without projection dephasing (Fig. 3a) and with projection dephasing $=0.24 \pi / \mathrm{mm}$ (Fig. 3c). Associated flow-noncompensated images (Figs. $3 \mathrm{~b}$ and $3 \mathrm{~d}$ ) are shown to the left of each angiogram for anatomical reference. Conspicuity of the vessels is enhanced by projection dephasing (SNR improvement by a factor of 5); however, as expected, vessel intensity is dependent on its position along the projection axis. The diameter of the large vessels midneck is approximately $1.4 \mathrm{~mm}$. Projection dephasing of $0.24 \pi / \mathrm{mm}$ results in a phase twist of $0.34 \pi$ across this vessel diameter which is well below the $2 \pi$ 

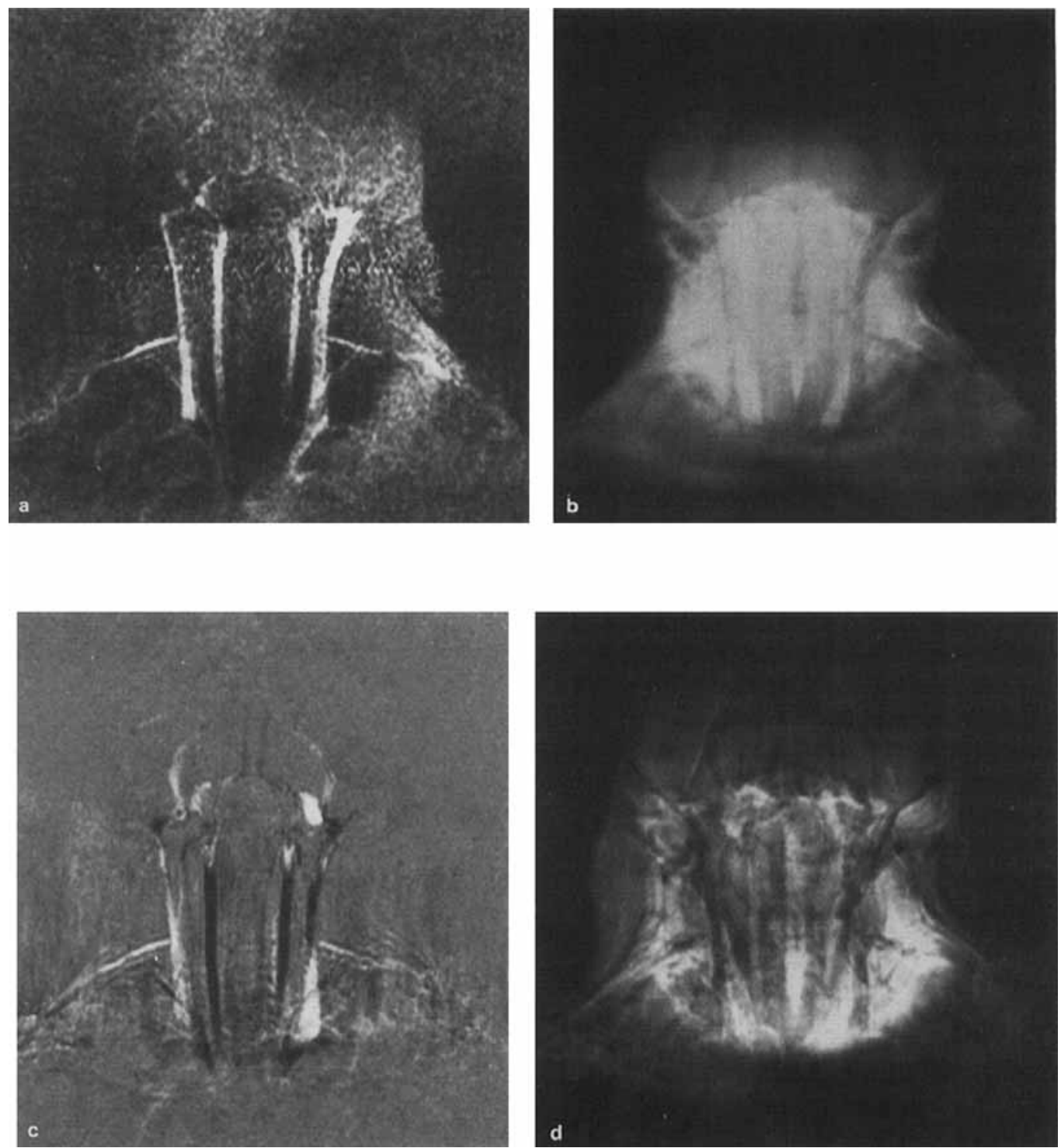

FiG. 3. (a) Magnitude difference angiogram of rat neck and (b) associated conventional projection image acquired without projection dephasing. (c) Magnitude difference and (d) corresponding projection image acquired with $0.24 \pi / \mathrm{mm}$ projection dephasing. Acquisitions were nongated, TR $=1000 \mathrm{~ms}$, TE $=20 \mathrm{~ms}$, two excitations, $\mathrm{FOV}=80 \mathrm{~mm}$.

limit where the flow signal is completely dephased. Figure 4 shows results of phase correction and complex subtraction applied to the same data as that in Fig. 3. Straightforward complex subtraction angiograms are shown on the left (Figs. 4a and $4 \mathrm{c}$ ) with the associated phase-corrected versions on the right (Figs. $4 \mathrm{~b}$ and $4 \mathrm{c}$ ). While position-dependent intensity modulations due to projection dephasing are removed, phase errors result in incomplete static signal subtraction in the noncorrected images. 

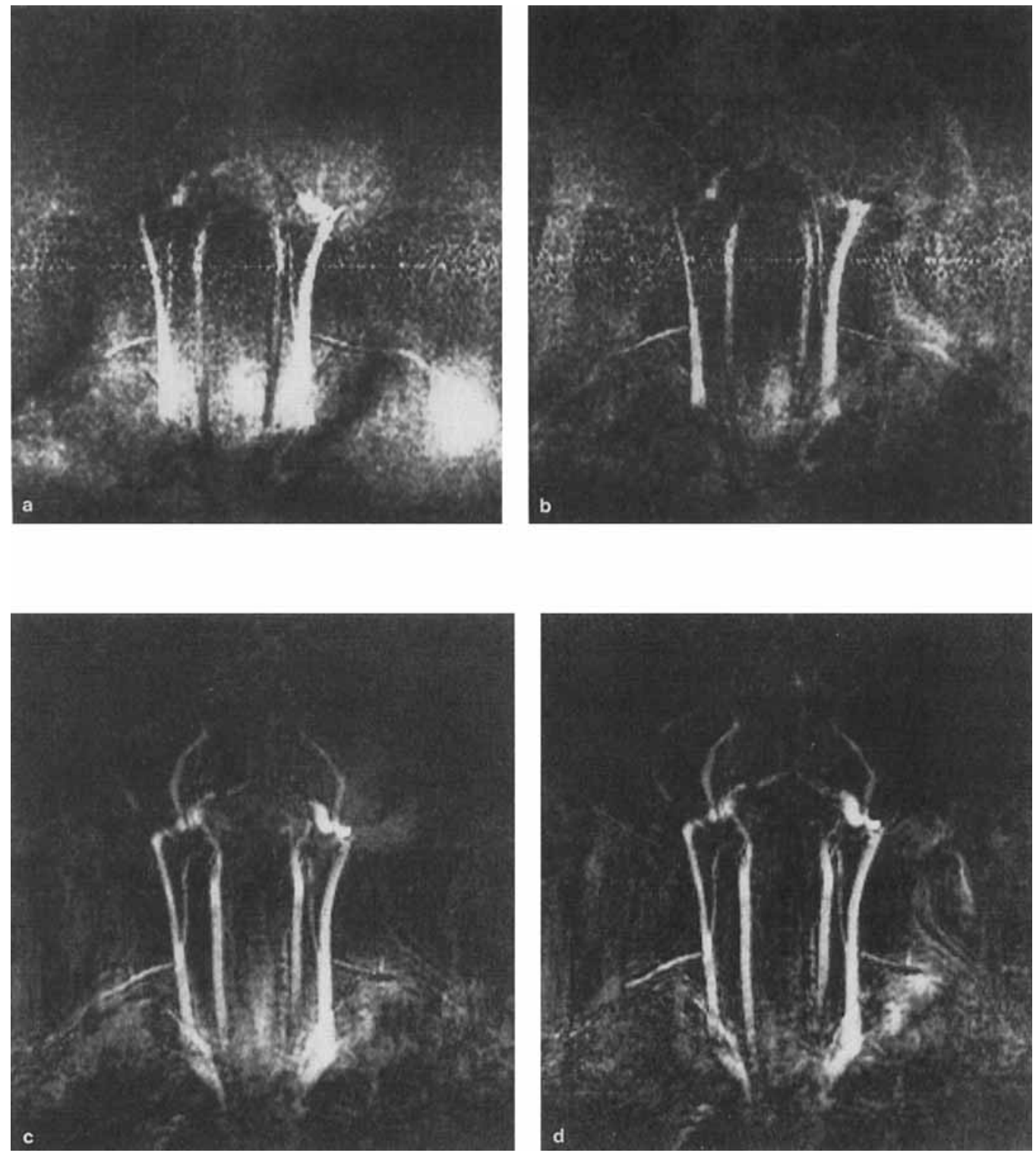

FiG. 4. Images obtained via complex subtraction of data sets used in Fig. 3, (a) prior to phase correction and (b) after phase correction of the nonprojection dephased data. Corresponding results obtained (c) without phase correction and (d) phase correction of the $0.24 \pi / \mathrm{mm}$ projection dephased data of Fig. 3 .

Comparing Figs. $4 \mathrm{a}$ and $4 \mathrm{c}$, it is interesting to note that projection dephasing alone helps reduce much of the phase error contamination of the difference image. When phase errors are large substantial improvements are realized via phase correction as evident by comparison of Figs. $4 \mathrm{a}$ and $4 \mathrm{~b}$. Conversely, only minor phase correction improvement is available when phase errors are small as apparent in Figs. $4 \mathrm{c}$ and $4 \mathrm{~d}$. 

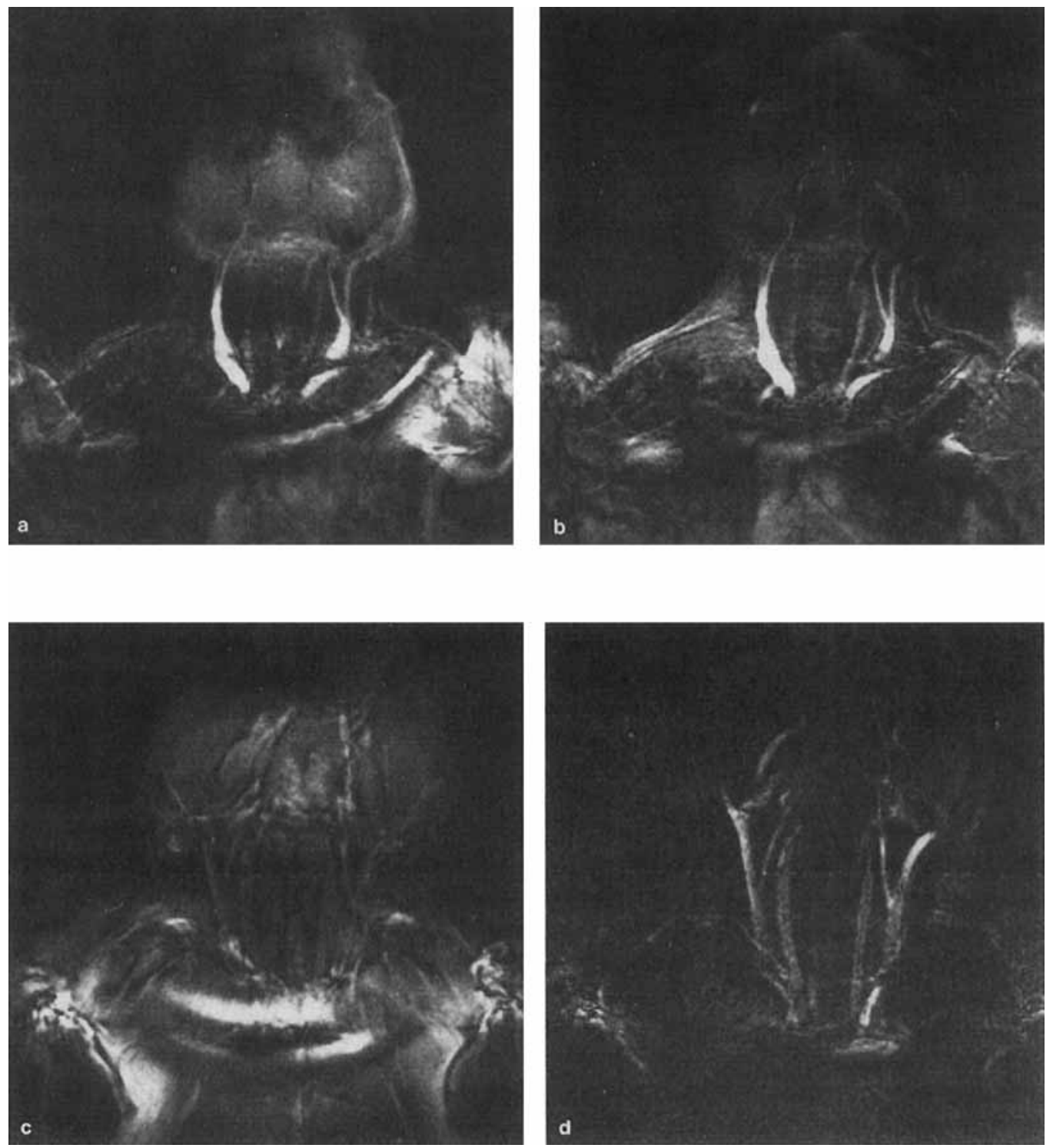

FIG. 5. Images from different animals: (a) complex difference image prior to phase correction and (b) after phase correction of $0.12 \pi / \mathrm{mm}$ projection dephased data. (c) Complex difference image prior to phase correction and (d) after phase correction of $0.24 \pi / \mathrm{mm}$ projection dephased data. Acquisitions were nongated, $\mathrm{TR}=500 \mathrm{~ms}, \mathrm{TE}=20 \mathrm{~ms}$, two excitations, $\mathrm{FOV}=80 \mathrm{~mm}$.

Note that some phase errors persist. Also, misregistration artifacts due object motion or eddy current differences during signal sampling are not removable by this scheme.

Figure 5 displays two more angiogram pairs differing in degree of phase error severity. The images in Figs. $5 \mathrm{a}$ and $5 \mathrm{~b}$ were collected with $0.12 \pi / \mathrm{mm}$ projection dephasing and those of the $5 \mathrm{c}-5 \mathrm{~d}$ pair were collected with $0.24 \pi / \mathrm{mm}$ projection dephasing. 
As noted above, slowly varying phase differences are nearly completely removed by the correction process, whereas misregistration or amplitude differences remain problematic. Most of the residual static tissue intensity in Fig. $5 \mathrm{~b}$ originates from the latter class. In contrast, the main source of static intensity in Fig. $5 \mathrm{c}$ is a large, but smoothly varying phase error propagating vertically through the image. This error is completely removed in Fig. 5d.

In summary, this paper addresses two main technical impediments to MR angiography: static signal limiting flow sensitivity and phase errors resulting from imperfect sequence execution. Static signals, even if ultimately subtracted, usually dominate receiver gain settings and consequently limit signal to noise of the subtraction angiogram. Means to reduce static signal prior to signal digitization include flow-selective excitation $(15,16)$ and projection dephasing $(10)$. Flow-selective excitation via combinations of $\mathrm{rf}$ and gradient pulses which restore static signal to the longitudinal direction may ultimately provide the greatest static signal suppression. However, since flow signal amplitude is dependent on flow speed, the flow-selective excitation technique is expected to have limited speed dynamic range. In addition, successful restoration of static signal to the longitudinal direction is dependent on static magnetic and rf field homogeneities, and accurate gradient pulse execution.

Because of its simplicity and compatibility with our objective to produce angiograms which are largely independent of flow speed we have incorporated projection dephasing. A notable drawback of projection dephasing is that the signal of superimposed vessels may interfere producing an apparent signal void at their intersection. A second potential drawback of projection dephasing is that complex subtraction of data is essential. This renders the angiogram susceptible to incomplete subtraction of static signal resulting from miscellaneous phase errors. The phase errors are usually due to poor sequence tuning or hardware limitations such as eddy currents. The severity and source of phase errors are system dependent. On our unit, eddy currents are the main source of error forcing us to limit the FOV to "well-behaved" regions in the magnet and to take special care in gradient waveform design. As long as the end product of the error is a smoothly spatially varying multiplicative phase function, simple phase correction schemes such as the method presented here are valuable. Thus far we have found the combination of projection-dephased high-resolution data and nonprojection-dephased low-resolution phase correction files to yield the best projection MR angiograms.

Others have presented a phase correction technique based on Chebyshev polynomial expansion of image data (12). While we have not implemented this approach for comparison to our technique, we expect that the results would be similar since both techniques strive to isolate the slowly varying phase component as the phase error.

\section{ACKNOWLEDGMENTS}

This work is supported by PHS Grant 5 R23 HL36105-02 awarded by the National Heart Lung and Blood Institute DHHS. The authors thank Jane Mitchell for manuscript preparation.

\section{REFERENCES}

1. J. P. FelmLeE AND R. L. EhMAN, Radiology 164, 559 (1987).

2. E. M. HAACKE AND G. W. LENZ, Amer. J. Roentgenol. 148, 1251 (1987). 
3. L. AXel, A. Shimakawa, and J. R. MacFall, Magn. Reson. Imaging 4, 199 (1986).

4. F. W. Wehrli, A. Shimakawa, G. T. GullberG, and J. R. MacFall, Radiology 160, 781 (1986).

5. D. A. Feinberg, L. Crooks, J. Hoenninger III, M. Arakawa, and J. Watts, Radiology 153, 177 (1984).

6. P. R. MORAN, Magn. Reson. Imaging 1, 197 (1982).

7. P. van DiJK, J. Comput. Assist. Tomogr. 8, 429 (1984).

8. D. J. Bryant, J. A. Payne, D. N. Firmin, and D. B. Longmore, J. Comput. Assist. Tomogr. 8, 588 (1984).

9. V. J. WedeEn, B. R. Rosen, R. BuXton, AND T. J. Brady, Magn. Reson. Med. 3, 226 (1986).

10. C. L. DUMOULIN AND H. R. HART, Radiology 161, 717 (1986).

11. L. Axel and D. Morton, J. Comput. Assist. Tomogr. 11, 31 (1987).

12. T. L. Chenevert, Med. Phys. 13, 177 (1986).

13. P. M. Pattany, R. Marino, and J. M. MCNally, Magn. Reson. Imaging 4, 154 (1986).

14. Q. S. Xiang AND O. Nalciolglu, "Fifth Annual Meeting of the Society of Magnetic Resonance in Medicine," p. 100, 1986.

15. P. R. Moran, D. SAloner, AND B. M. W. TSUI, IEEE Trans. Med. Imaging MI-6, 141 (1987).

16. J. Pauly, D. Nishimura, AND A. Macovski, "Sixth Annual Meeting of the Society of Magnetic Resonance in Medicine," p. 27, 1987.

17. J. J. E. IN DEN KLEEF AND J. P. GROEN, "Sixth Annual Meeting of the Society of Magnetic Resonance in Medicine," p. 29, 1987. 